\title{
FILOSOFIA ASSISTENCIAL DE UMA MATERNIDADE-ESCOLA: FATORES ASSOCIADOS À SATISFAÇÃO DAS MULHERES USUÁRIAS
}

\author{
Odaléa Maria Brüggemann ${ }^{1}$, Marisa Monticelli², Camilla Furtado ${ }^{3}$, Camila Mariano Fernandes ${ }^{4}$, Fabiane \\ Nunes Lemos ${ }^{5}$, Michele Edianez Gayeski ${ }^{6}$
}

\footnotetext{
${ }^{1}$ Doutora em Tocoginecologia. Docente do Programa de Pós-graduação em Enfermagem (PEN) da Universidade Federal de Santa Catarina (UFSC). Pesquisadora CNPq. Santa Catarina, Brasil. E-mail: odalea@ccs.ufsc.br

${ }^{2}$ Doutora em Enfermagem. Docente do PEN/UFSC. Santa Catarina, Brasil. E-mail: marisa@ccs.ufsc.br

${ }^{3}$ Acadêmica de Graduação em Enfermagem da UFSC. Bolsista PIBIC/CNPq 2009-2010. Santa Catarina, Brasil. E-mail: milla. furtado@hotmail.com

${ }^{4}$ Enfermeira Residente - Residência Multiprofissional em Saúde da Família - UFSC/Ministério da Saúde. Santa Catarina, Brasil E-mail: camila-mf@hotmail.com

${ }^{5}$ Acadêmica de Graduação em Enfermagem da UFSC. Santa Catarina, Brasil. E-mail: byane_@hotmail.com

${ }^{6}$ Doutoranda do PEN/UFSC. Enfermeira da Hospital Universitário da UFSC. Santa Catarina, Brasil. Email: michelegayeski@ hotmail.com
}

RESUMO: Estudo transversal que avaliou a filosofia de uma maternidade, a partir da satisfação das mulheres com o atendimento recebido na admissão obstétrica, no trabalho de parto/parto e no puerpério. Os dados foram coletados com 310 mulheres e analisados pelo teste qui-quadrado, exato de Fisher, Odds Ratio (IC 95\%) e regressão logística. A satisfação na admissão esteve estatisticamente associada ao fato dos profissionais e acadêmicos se apresentarem, darem as orientações solicitadas e fornecerem apoio emocional; no trabalho de parto/parto esteve associada com privacidade, apoio emocional e recebimento de informações; e no puerpério, com apoio emocional e recebimento de informações. Chance de insatisfação ocorreu quando a mulher não recebeu apoio emocional na admissão $(5,70$; IC 95\%: 1,88; 17,31) e não obteve informações no trabalho de parto/parto $(6,53 ;$ IC 95\%: 1,97; 21,64). A concretização de uma filosofia assistencial que atenda as necessidades das usuárias contribui para a satisfação com o atendimento, desde a internação até a alta. DESCRITORES: Serviços de saúde materna. Avaliação de serviços de saúde. Satisfação dos consumidores. Parto humanizado.

\section{PHILOSOPHY OF CARE IN A TEACHING MATERNITY HOSPITAL: FACTORS ASSOCIATED TO FEMALE USERS SATISFACTION}

\begin{abstract}
This cross-sectional study assessed the philosophy of a maternity based on women users satisfaction factors concerning the services received during their admission, labor/delivery, and puerperium. Data was collected from 310 women and analyzed by chi-square test, Fisher exact test, Odds Ratio (95\%), and logistic regression. Satisfaction at admission was statistically associated with the fact that professionals and academics introduced themselves, gave the orientation requested, and provided emotional support. In labor/ delivery satisfaction was associated with privacy, emotional support, and receiving information. In the postpartum period, satisfaction was associated with emotional support and receiving information. The chance of dissatisfaction occurred when the woman was not emotionally supported at admission (5.70; 95\% CI: 1.88; 17.31) and didn't obtain information during labor/delivery $(6.53 ;$ CI 95\%: 1.97; 21.64). The implementation of a care philosophy that meets user needs contribute to satisfaction with the service provided from admission to discharge. DESCRIPTORS: Maternal health services. Health services evaluation. Consumer satisfaction. Humanizing delivery.
\end{abstract}

\section{LA FILOSOFÍA DE ATENCIÓN DE UNA MATERNIDAD ESCUELA: FATORES ASOCIADOS CON LA SATISFACCIÓN DE LAS MUJERES USUARIAS}

RESUMEN: Es un estudio transversal que evaluó la filosofía de una maternidad, a partir de la satisfación con la atención ricibieda em la admisión, el trabajo de parto y el posparto. La recolección de los datos se hizo con 310 mujeres y se analizaron mediante la prueba de chi-cuadrado, prueba exacta de Fisher, Odds Ratio (IC 95\%) y regresión logística. La satisfacción por la admisión se asoció estatisticamente con el hecho de que los profesionales y académicos estuvieron presentes, dieron las orientaciones solicitadas y el apoyo emocional. En el trabajo de parto y el parto se asoció con la intimidad, apoyo emocional y la información que recibieron, y en el puerperio, con el apoyo emocional y recibir informaciones. La insatisfacción se produjo cuando la mujer no recibió el apoyo emocional al ingreso $(5,70$, IC 95\%: 1.88; 17.31) y no recibió información sobre el trabajo de parto y el parto $(6,53$, IC 95\%: 1,97; 21,64). La aplicación de una filosofía de atención que responda a las necesidades de las usuarias contribuye a la satisfacción con los servicios, desde la hospitalización hasta el alta.

DESCRIPTORES: Servicios de salud materna. Evaluación de servicios de salud. Satisfacción de los consumidores. Parto humanizado. 


\section{INTRODUÇÃO}

Qualquer política ou filosofia de atenção à saúde somente ocorre efetivamente quando seus princípios são materializados, mas isso pressupõe aderência dos profissionais a tais propostas, a fim de que as mesmas sejam colocadas em prática, em benefício dos usuários. O problema desta pesquisa está voltado para a aplicabilidade da filosofia assistencial da maternidade do Hospital Universitário (HU) da Universidade Federal de Santa Catarina (UFSC). Esta investigação faz parte de um macroprojeto, sendo que a filosofia já foi avaliada pelos profissionais e acadêmicos que atuam na instituição. ${ }^{1} \mathrm{O}$ relato, agora, se direciona para as usuárias, ou seja, as mulheres que deram à luz na maternidade.

A construção de um modelo assistencial para dar sustentação à implantação da maternidade iniciou em 1980 e se estendeu até 1995, quando foi inaugurada. Nesse intervalo, um grupo de profissionais estabeleceu que o modelo de atenção obstétrico e neonatal a ser adotado, deveria respeitar as recomendações da Organização Mundial da Saúde ${ }^{2}$ e os fundamentos das evidências científicas na área da saúde. ${ }^{3}$ Isso culminou na elaboração de um conjunto de 12 princípios filosóficos, que passou a ser reconhecido como "a filosofia da maternidade". ${ }^{4}$

Tal filosofia contempla três abordagens: $1^{\text {a) }}$ ) Relacionada com os aspectos gerais da assistência. Engloba os seguintes princípios: A atenção à saúde da mulher visa o ciclo gravídico-puerperal, considerando a gravidez como um processo e não como um evento; A parturiente não deixará de ser assistida em face de problemas burocráticos, sendo que as rotinas deverão ser flexíveis; A mulher deve permanecer internada o tempo suficiente para que sejam atendidas suas necessidades assistenciais e de educação em saúde. $2^{a}$ ) Relacionada com a integração entre os profissionais e os serviços, envolvendo os seguintes princípios: A equipe interdisciplinar que presta assistência à mulher/ recém-nascido/família deve atuar de forma integrada, visando um atendimento adequado; As atividades de ensino, pesquisa e extensão realizadas pela equipe interdisciplinar devem refletir atitudes de respeito ao ser humano e reverter em benefício de melhor assistência; A equipe deve exercer papel atuante na educação da mulher/acompanhante e grupo familiar, com vistas ao preparo e adaptação do aleitamento materno, desenvolvimento da confiança e capacidade de cuidar do filho, execução de cuidados básicos de saúde e planejamento familiar; Todo pessoal deve ter qualificação, treinamento e supervisão contínua e específica para prestação da assistência a que tem direito a mãe, o recém-nascido e a família; e O desenvolvimento de atividades será de forma integrada quanto às unidades que operam na maternidade, ou que com ela se relacionem. $3^{\text {a) }}$ Relacionada aos direitos dos usuários. Esta abordagem engloba os princípios: É direito de toda mulher/Recém-Nascido (RN)/ família, no processo de gravidez, parto e puerpério, receber atendimento personalizado que garanta uma assistência adequada, nos aspectos biológicos, sociais, psicológicos e espirituais; $\mathrm{Na}$ atenção à saúde da mãe, RN e família, na gravidez, parto e puerpério, se considera a importância do papel do pai, sua presença e participação; e O sistema de alojamento conjunto facilita a criação e aprofundamento de laços mãe-RN-família, favorecendo a vinculação afetiva, a compreensão do processo de crescimento e desenvolvimento, a participação ativa e a educação para a saúde dos elementos mencionados.

Para garantir a aplicabilidade dessa filosofia, um grupo multiprofissional ligado à Direção Geral da instituição, desenvolve atividades e discussões e apoia estudos ${ }^{1,5}$ no sentido de avaliar se a mesma está sendo implementada pelos profissionais e acadêmicos em todos os setores da maternidade. Apesar disso, ainda permanece uma importante lacuna, relacionada com a perspectiva das mulheres usuárias. Esta avaliação é necessária não apenas para aumentar o escopo analítico da verificação da aplicação da filosofia, mas também para observar se essas mulheres estão sendo beneficiadas com a política assistencial adotada.

Assim, o objetivo desta investigação foi avaliar a implementação da filosofia da maternidade do HU/UFSC, a partir da satisfação das mulheres com o atendimento recebido na admissão obstétrica, no trabalho de parto/parto e no puerpério. Essa investigação, sob a ótica das usuárias, teve o propósito de analisar, de modo indireto, como os princípios filosóficos que norteiam a assistência na referida maternidade são implementados no cotidiano institucional. Neste contexto, tomou-se como preditor da satisfação, a qualidade do cuidado prestado ${ }^{6}$, contemplando, o tipo de apoio recebido, a interação/comunicação com os profissionais, as características ambientais da maternidade e se as práticas obstétricas desenvolvidas estavam amparadas em evidências científicas.

\section{MÉTODO}

Trata-se de um estudo transversal, que foi desenvolvido na maternidade do HU/UFSC, que é um hospital-escola de médio porte e presta aten- 
dimento de média à alta complexidade, atendendo exclusivamente pelo Sistema Único de Saúde. A maternidade engloba a Triagem obstétrica, com duas salas de exame e uma de observação; o Centro Obstétrico, com quatro leitos de pré-parto, um leito de observação e duas salas de parto/cirúrgicas; e o Alojamento Conjunto, com 22 leitos e berços.

O protocolo assistencial preconiza a adoção de práticas com base nas evidências científicas. Assim, entre outras, não é rotina realizar o enema, restrição de alimentos líquidos ou sólidos e nem episiotomia; a tricotomia é realizada apenas no local da incisão cirúrgica, nos casos de cesariana; é propiciada à parturiente a escolha da posição no parto normal (vertical ou horizontal); é permitida e estimulada a presença de um acompanhante de escolha desde a admissão obstétrica até a alta hospitalar; e são oferecidos métodos não farmacológicos para alívio da dor, como técnicas de banho, massagem, uso da bola e do "cavalinho". O tempo de internação é estipulado de acordo com as necessidades específicas das puérperas e dos RNs. ${ }^{4}$

Os dados foram coletados no período de outubro de 2008 a dezembro de 2009. A amostra foi constituída por mulheres internadas no Alojamento Conjunto. O tamanho da amostra foi calculado com base na realização de 1.600 partos anuais realizados no HU. Estimou-se a satisfação com o atendimento recebido em $50 \%$, intervalo de confiança de $95 \%$ e erro máximo de $5 \%$, resultando em um total de 310 mulheres. Os critérios de inclusão foram ter sido atendida durante o trabalho de parto e ter tido feto vivo, independente da via de parto. Não foram incluídas as submetidas à cesariana eletiva e/ou as mulheres cujo RN foi a óbito. Esses critérios foram assim estabelecidos por considerar-se que poderiam influenciar nas respostas relativas à satisfação com a assistência prestada.

Para a coleta de dados aplicou-se um formulário por meio de entrevistas. Este instrumento continha perguntas referentes às atividades assistenciais desenvolvidas junto às usuárias na admissão, durante o trabalho de parto/ parto, assim como no puerpério. A intencionalidade foi contemplar os princípios contidos nas três abordagens que integralizam a filosofia da maternidade, ou seja, relacionados com os aspectos gerais da assistência, com a integração entre os profissionais e os serviços, e com os direitos dos usuários. A satisfação foi avaliada tomando-se como referência as respostas obtidas a partir de uma seqüência de cinco símbolos com expressões faciais, mostrados à usuária, para que ela apontasse qual correspon- dia à forma como se sentiu (muito insatisfeita, insatisfeita, indiferente, bem satisfeita ou muito satisfeita) com o atendimento recebido na admissão obstétrica, durante o trabalho de parto/parto e no puerpério.

O formulário foi testado com cinco mulheres, sendo reformuladas as questões que não estavam totalmente compreensíveis, com a intenção de obter informações fidedignas. As entrevistas ocorreram nas primeiras 12-24 horas pós-parto, de forma sequencial, com aquelas que atenderam os critérios de inclusão, até completar o tamanho amostral, sendo que não houve recusa. No processo de organização e análise dos dados, todos os questionários foram revisados, codificados e digitados no programa EPI INFO - versão 2002.

Realizou-se análise estatística descritiva (distribuição absoluta e relativa) dos dados e a satisfação das mulheres foi quantificada através da escala tipo Likerte considerado como indicativo de satisfação as respostas bem satisfeita e muito satisfeita. ${ }^{7-8}$ Cada um dos fatores foi estudado quanto à satisfação, através do teste de qui-quadrado ou exato de Fisher. A razão de chance de cada um dos fatores em relação à satisfação foi mensurada através do cálculo do Odds Ratio e respectivos intervalos de confiança a $95 \%$, para a satisfação com o atendimento recebido na admissão, durante o trabalho de parto/parto e no puerpério. Os valores do Odds Rattio também foram calculados para verificar os fatores relacionados com a insatisfação, ou seja, os princípios filosóficos que não estavam sendo colocados em prática na assistência prestada a essas usuárias.

A fim de verificar, dentre os fatores estudados (e suas inter-relações), os que apresentavam maior relação com as variáveis dependentes, foi realizada a análise múltipla dos fatores, através de regressão logística, com critério de seleção de variáveis stepwise. Esta análise foi realizada considerando a variável dependente satisfação, e realizada de acordo com as variáveis aferidas segundo dois diferentes grupos. O primeiro, referente à admissão obstétrica, composto por todas as variáveis apresentadas na tabela 2, e o segundo, referente ao trabalho de parto/ parto, cujas variáveis encontram-se na tabela 3.

A pesquisa seguiu a Declaração de Helsinque e a Resolução 196/96 do Conselho Nacional de Saúde. Todas as entrevistadas assinaram o Termo de Consentimento Livre e Esclarecido. O projeto foi aprovado pelo Comitê de Ética em Pesquisa da UFSC - protocolo $n^{\circ} 263 / 07$. 


\section{RESULTADOS}

Quanto às características sociodemográficas, a mediana da idade esteve em torno de 25 anos. A maioria delas era casada ou em união consensual $(84,9 \%)$, branca $(75,2 \%)$, católica $(66,4 \%)$ e com pouca escolaridade. Quanto às características obstétricas, 51,3\% eram primíparas e 48,7\% multíparas; $70,3 \%$ realizaram seis consultas ou mais de pré-natal; $55,5 \%$ pariram na posição vertical e $11,6 \%$, na posição horizontal, totalizando $67,4 \%$ de partos vaginais; a taxa de cesariana foi de $32,6 \%$. Além disso, a imensa maioria $(81,0 \%)$ não participou de grupo de gestantes (Tabela 1).

Tabela 1 - Características sociodemográficas e obstétricas das mulheres usuárias. FlorianópolisSC, $2009(n=310)$

\begin{tabular}{lrr}
\hline Variáveis & $\mathbf{n}$ & \multicolumn{1}{c}{$\%$} \\
\hline Idade (mediana em anos) & $25(14$ & $-43)$ \\
Estado marital & & \\
Casada/União consensual & 263 & 84,9 \\
Solteira/Separada/Viúva & 47 & 15,1 \\
Cor & & \\
Branca & 233 & 75,2 \\
Negra & 43 & 13,9 \\
Outra & 34 & 11,0 \\
Ocupação & & \\
Atividade remunerada & 149 & 48,0 \\
Do lar & 123 & 39,8 \\
Estudante & 29 & 9,3 \\
Desempregada & 9 & 2,9 \\
Religião & & \\
Católica & 206 & 66,4 \\
Evangélica & 42 & 13,5 \\
Nenhuma & 36 & 11,6 \\
Outra & 26 & 8,4 \\
Escolaridade & & \\
Ensino fundamental incompleto & 58 & 18,7 \\
Ensino fundamental completo & 129 & 41,6 \\
Ensino médio completo & 115 & 37,0 \\
Ensino superior completo & 6 & 1,9 \\
Nenhuma & 2 & 0,6 \\
Paridade & & \\
Primípara & 159 & 51,3 \\
Multípara & 151 & 48,7 \\
Número de consultas pré-natal & & \\
$\geq 6$ consultas & 218 & 70,3 \\
< 6 consultas & 89 & 28,7 \\
& & \\
& & \\
& &
\end{tabular}

Nenhuma

3

Participação em grupo de gestantes

Não

25181,0

Sim

$59 \quad 19,0$

Tipo de parto atual

Vaginal na posição vertical

$172 \quad 55,5$

Cesariana

10132,6

Vaginal na posição horizontal

$36 \quad 11,6$

Vaginal à fórceps

$1 \quad 0,3$

A implementação dos princípios das três abordagens que integralizam a filosofia da maternidade encontra-se expressa nos resultados sobre o atendimento recebido pelas mulheres usuárias na admissão obstétrica (Tabela 2), durante o trabalho de parto/parto (Tabela 3) e no puerpério (Tabela 4).

A avaliação que essas mulheres fizeram sobre o atendimento recebido na admissão obstétrica revela que a maioria sabia identificar quem era o profissional que a tinha recebido no momento da internação. A enfermeira foi referida com um maior percentual $(61,9 \%)$, seguida pelo médico (55,5\%). As mulheres também relataram que as pessoas que prestaram atendimento se apresentaram $(89,0 \%)$, encontravam-se capacitadas $(95,2 \%)$ e interessadas em prestar assistência $(95,8 \%)$ e deram as informações que foram solicitadas $(87,8 \%)$, inclusive sobre o motivo obstétrico que as levou a permanecerem internadas $(88,4 \%)$, assim como, que receberam apoio emocional durante o momento inicial da internação $(84,2 \%)$ Quanto às práticas obstétricas, a quase totalidade das mulheres não foi submetida à enema $(99,0 \%)$. A maioria não realizou tricotomia $(79,7 \%)$ e teve um acompanhante no momento da internação $(95,5 \%)$ (Tabela 2).

Com relação à satisfação com o atendimento recebido na admissão obstétrica, diversos fatores apresentaram associação significativa como o fato dos profissionais/acadêmicos se apresentarem $(p=0,0014)$, estarem capacitados e interessados para atender $(p<0,0001)$, darem as orientações solicitadas $(p<0,0001)$, informarem o motivo da internação $(p<0,0001)$ e oferecerem apoio emocional $(\mathrm{p}<0,0001)$. A não realização dessas ações foram considerados fatores que aumentaram a chance de insatisfação das mulheres, em diferentes magnitudes, avaliadas através da razão de chance Odds Ratio (com intervalos de confiança significativos) (Tabela 2). 
Tabela 2 - Atendimento na admissão obstétrica e satisfação segundo as mulheres usuárias. Florianópolis-SC, 2009 (n=310)

\begin{tabular}{|c|c|c|c|c|c|c|}
\hline Variáveis & $\begin{array}{c}\text { Total } \\
310 \\
\mathrm{n}(\%)\end{array}$ & $\begin{array}{c}\text { Satisfeita } \\
278(89,7) \\
\text { n(\%) }\end{array}$ & $\begin{array}{c}\text { Insatisfeita } \\
32(10,3) \\
n(\%)\end{array}$ & Valor $p$ & OR(IC95\%) & $\begin{array}{l}\text { OR múltiplo§ } \\
\text { (IC 95\%) }\end{array}$ \\
\hline \multicolumn{7}{|l|}{ Pessoas que atenderam ${ }^{*}$} \\
\hline Enfermeira & $192(61,9)$ & $172(89,6)$ & $20(10,4)$ & 0,9446 & $1,03(0,48 ; 2,19)$ & $\mathrm{ns}$ \\
\hline Médico & $171(55,5)$ & $154(90,1)$ & $17(9,9)$ & 0,8068 & $0,91(0,44 ; 1,90)$ & ns \\
\hline Acadêmico de medicina & $34(10,9)$ & $32(94,1)$ & $2(5,9)$ & $0,5521 \ddagger$ & $0,51(0,12 ; 2,25)$ & ns \\
\hline Auxiliar/técnico de enfermagem & $30(9,6)$ & $29(96,7)$ & $1(3,3)$ & $0,3383 \ddagger$ & $0,28(0,04 ; 2,11)$ & ns \\
\hline Acadêmico de enfermagem & $6(1,9)$ & $1(100,0)$ & $0(0,0)$ & $1,0000 \ddagger$ & $0,64(0,04 ; 11,71)$ & ns \\
\hline $\begin{array}{l}\text { Profissionais/acadêmicos se } \\
\text { apresentaram }\end{array}$ & & & & $0,0014 \ddagger$ & & \\
\hline Sim & $276(89,0)$ & $254(92,0)$ & $22(8,0)$ & & 1,00 & \\
\hline Não & $26(8,40)$ & $19(73,1)$ & $7(26,9)$ & & $4,25(1,61 ; 11,22)$ & ns \\
\hline Não lembra & $89(2,6)$ & $5(62,5)$ & $3(37,5)$ & & $6,93(1,55 ; 30,93)$ & ns \\
\hline $\begin{array}{l}\text { Profissionais/acadêmicos } \\
\text { capacitados para atender }\end{array}$ & & & & $<0,0001$ & & \\
\hline Sim & $295(95,2)$ & $272(92,2)$ & $23(7,8)$ & & 1,00 & 1,00 \\
\hline Não & $15(4,8)$ & $6(40,0)$ & $9(60,0)$ & & $17,74(5,8 ; 54,22)$ & $15,10(4,00 ; 59,36)$ \\
\hline $\begin{array}{l}\text { Profissionais/acadêmicos } \\
\text { interessados em atender }\end{array}$ & & & & $<0,0001$ & & \\
\hline Sim & $297(95,8)$ & $271(91,2)$ & $26(8,8)$ & & 1,00 & \\
\hline Não & $13(4,2)$ & $7(53,8)$ & $6(46,2)$ & & $8,93(2,79 ; 28,57)$ & ns \\
\hline $\begin{array}{l}\text { Profissionais/acadêmicos deram } \\
\text { as informações solicitadas }\end{array}$ & & & & $<0,0001 \ddagger$ & & \\
\hline Sim & $272(87,8)$ & $252(92,6)$ & $20(7,4)$ & & 1,00 & \\
\hline Algumas vezes & $20(6,4)$ & $10(50,0)$ & $10(50,0)$ & & $12,60(4,69 ; 33,83)$ & $8,78(2,77 ; 27,83)$ \\
\hline Não solicitou & $15(4,8)$ & $14(93,3)$ & $1(6,7)$ & & $0,90(0,11 ; 7,20)$ & $0,77(0,09 ; 6,84)$ \\
\hline Não lembra & $3(1,0)$ & $2(66,7)$ & $1(33,3)$ & & $6,30(0,55 ; 72,52)$ & $2,73(0,01 ; 126,13)$ \\
\hline $\begin{array}{l}\text { Recebeu informação sobre o } \\
\text { motivo da internação }\end{array}$ & & & & $<0,0001 \ddagger$ & & \\
\hline Sim & $274(88,4)$ & $252(92,0)$ & $22(8,0)$ & & 1,00 & \\
\hline Não & $25(8,1)$ & $19(76,0)$ & $6(24,0)$ & & $3,62(1,31 ; 9,99)$ & ns \\
\hline Não lembra & $11(3,5)$ & $7(63,6)$ & $4(36,4)$ & & $6,55(1,78 ; 24,10)$ & $\mathrm{ns}$ \\
\hline $\begin{array}{l}\text { Profissionais/acadêmicos } \\
\text { ofereceram apoio emocional }\end{array}$ & & & & $<0,0001$ & & \\
\hline Sim & $261(84,2)$ & $246(94,3)$ & $15(5,7)$ & & 1,00 & 1,00 \\
\hline Não & $26(8,4)$ & $17(65,4)$ & $9(34,6)$ & & $8,68(3,32 ; 22,71)$ & $5,70(1,88 ; 17,31)$ \\
\hline Em parte & $23(7,4)$ & $15(65,2)$ & $8(34,8)$ & & $8,75(3,20 ; 23,87)$ & $4,20(1,24 ; 14,16)$ \\
\hline Realizaram enema & & & & $0,2796 \ddagger$ & & \\
\hline Sim & $3(1,0)$ & $2(66,7)$ & $1(33,3)$ & & 1,00 & ns \\
\hline Não & $307(99,0)$ & $276(89,9)$ & $31(10,1)$ & & $0,22(0,02 ; 2,55)$ & ns \\
\hline Realizaram tricotomia $^{\dagger}$ & & & & 0,1048 & & \\
\hline Sim & $63(20,3)$ & $53(84,1)$ & $10(15,9)$ & & $1,93(0,86 ; 4,32)$ & $2,79(1,09 ; 7,19)$ \\
\hline Não & $247(79,7)$ & $225(91,1)$ & $22(8,9)$ & & 1,00 & 1,00 \\
\hline $\begin{array}{l}\text { Teve acompanhante na sala de } \\
\text { admissão }\end{array}$ & & & & $0,1657 \ddagger$ & & \\
\hline Sim & $296(95,5)$ & $267(90,2)$ & $29(9,8)$ & & 1,00 & ns \\
\hline $\begin{array}{l}\text { Não quis/acompanhante não } \\
\text { disponível }\end{array}$ & $14(4,5)$ & $11(78,6)$ & $3(21,4)$ & & $2,51(0,66 ; 9,52)$ & ns \\
\hline
\end{tabular}

* Algumas mulheres foram atendidas por mais de uma pessoa e 55 mulheres não sabiam informar; ${ }^{\dagger}$ Procedimento realizado somente em cesarianas, quando necessário; Teste de qui-quadrado / ₹ Teste exato de Fisher; ${ }^{\S}$ Regressão logística múltipla com critério de seleção stepwise, sendo incluídas todas as variáveis presentes na tabela, exceto as com frequência n=0; ns=não significativo na analise múltipla. 
$\mathrm{Na}$ análise múltipla, observou-se maior chance de insatisfação quando a mulher foi atendida por profissionais/acadêmicos não capacitados $(15,10$; IC95\%: 4,00; 59,36), quando recebeu as informações solicitadas somente algumas vezes $(8,78$; IC95\%: 2,77; 27,83), quando não recebeu apoio emocional $(5,70$; IC95\%: 1,88; 17,31) ou quando recebeu de forma parcial $(4,20$; IC95\%: $1,24 ; 14,16)$. Entre as práticas realizadas, apenas a tricotomia aumentou a chance de insatisfação (2,79; IC95\%: 1,09; 7,19) (Tabela 2).

Quanto ao atendimento recebido durante o trabalho de parto/parto, expressiva parcela referiu que os profissionais/acadêmicos respeitaram a privacidade $(95,5 \%)$, ofereceram apoio emocional $(93,2 \%)$ e responderam aos questionamentos $(93,9 \%)$. Praticamente metade das usuárias recebeu alimentos sólidos ou líquidos (49,7\%); a maioria foi estimulada a deambular ou movimentar-se $(80,0 \%)$ e utilizou métodos não farmacológicos para alívio da dor (técnicas de banho, massagem e outras estratégias) $(69,0 \%)$. Durante o trabalho de parto, $57,7 \%$ das mulheres foram levadas para conhecer as salas de parto normal e cirúrgico e
42,3\% não foram, sendo que, destas últimas, 45,8\% não conheceram previamente, pois não houve tempo, em vista da rápida evolução do processo. Das que evoluíram para parto normal, 80,4\% tiveram respeitada a posição escolhida para parir. A quase totalidade pôde contar com a presença de acompanhante no Centro Obstétrico e teve contato imediato com o RN logo após o parto $(91,3 \%)$ (Tabela 3).

Na análise da satisfação das usuárias com o atendimento durante o trabalho de parto/parto, observou-se associação significativa no que se refere a ter a privacidade respeitada $(p=0,0449)$, receber apoio emocional $(p=0,0353)$ e receber as informações que foram solicitadas aos profissionais/acadêmicos $(p<0,0161)$. Destaca-se que a realização parcial dessas ações aumentou a chance de insatisfação em 5,47 vezes (IC95\%: 1,03; 29,16) com relação à privacidade e em 5,69 vezes (IC95\%: $1,40 ; 23,08)$ quanto ao apoio emocional. Não receber as informações solicitadas também aumentou a chance de insatisfação $(6,53 ;$ IC $95 \%: 1,97 ; 21,64)$, sendo esse resultado confirmado também pela análise múltipla (Tabela 3).

Tabela 3 - Atendimento durante o trabalho de parto/parto e satisfação segundo as mulheres usuárias. Florianópolis -SC, $2009(\mathrm{n}=310)$

(continua)

\begin{tabular}{|c|c|c|c|c|c|c|}
\hline Variáveis & $\begin{array}{c}\text { Total } \\
310 \\
\mathrm{n}(\%) \\
\end{array}$ & $\begin{array}{c}\text { Satisfeita } \\
290(93,5) \\
n(\%) \\
\end{array}$ & $\begin{array}{c}\text { Insatisfeita } \\
20(6,5) \\
n(\%) \\
\end{array}$ & Valor $p$ & OR(IC95\%) & $\begin{array}{l}\text { OR múltiplo§ } \\
\text { (IC 95\%) }\end{array}$ \\
\hline Respeitaram a privacidade & & & & $0,0449 \neq$ & & \\
\hline Sim & $296(95,5)$ & $279(94,3)$ & $17(5,7)$ & & 1,00 & \\
\hline Não & $6(1,4)$ & $5(83,3)$ & $1(16,7)$ & & $3,28(0,36 ; 29,69)$ & ns \\
\hline Em parte & $8(2,6)$ & $6(75,0)$ & $2(25,0)$ & & $5,47(1,03 ; 29,16)$ & ns \\
\hline $\begin{array}{l}\text { Profissionais/acadêmicos } \\
\text { ofereceram apoio emocional }\end{array}$ & & & & $0,0353^{\ddagger}$ & & \\
\hline Sim & $289(93,2)$ & $273(94,5)$ & $16(5,5)$ & & 1,00 & \\
\hline Não & $9(2,9)$ & $8(88,8)$ & $1(11,1)$ & & $2,13(0,25 ; 18,11)$ & ns \\
\hline Em parte & $12(3,9)$ & $9(75,0)$ & $3(25,0)$ & & $5,69(1,40 ; 23,08)$ & ns \\
\hline $\begin{array}{l}\text { Profissionais/acadêmicos deram } \\
\text { as informações solicitadas }\end{array}$ & & & & $0,0161^{\ddagger}$ & & \\
\hline Sim & $291(93,9)$ & $275(94,5)$ & $16(5,5)$ & & 1,00 & 1,00 \\
\hline Não & $4(1,3)$ & $4(100,0)$ & $0(0,00)$ & & $1,86(0,10 ; 35,94)$ & $1,86(0,10 ; 35,94)$ \\
\hline Em parte & $15(4,8)$ & $11(73,3)$ & $4(26,7)$ & & $6,53(1,97 ; 21,64)$ & $6,53(1,97 ; 21,64)$ \\
\hline $\begin{array}{l}\text { Ofereceram alimentos sólidos ou } \\
\text { líquidos }\end{array}$ & & & & 0,3708 & & \\
\hline Sim & $154(49,7)$ & $146(94,8)$ & $8(5,2)$ & & 1,00 & \\
\hline Não & $156(50,3)$ & $144(92,3)$ & $12(7,7)$ & & $1,52(0,60 ; 3,83)$ & ns \\
\hline $\begin{array}{l}\text { Foi estimulada para deambular } \\
\text { ou movimentar-se }\end{array}$ & & & & $0,7744^{\ddagger}$ & & \\
\hline Sim & $248(80,0)$ & $231(93,1)$ & $17(6,9)$ & & 1,00 & \\
\hline Não* & $62(20,0)$ & $59(95,2)$ & $3(4,8)$ & & $0,69(0,20 ; 2,44)$ & ns \\
\hline
\end{tabular}




\begin{tabular}{|c|c|c|c|c|c|c|}
\hline $\begin{array}{l}\text { Aplicaram métodos não } \\
\text { farmacológicos para alívio da dor }\end{array}$ & & & & 0,3664 & & \\
\hline Sim & $214(69,0)$ & $202(94,4)$ & $12(5,6)$ & & 1,00 & \\
\hline Não & $96(31,0)$ & $88(91,7)$ & $8(8,3)$ & & $1,53(0,60 ; 3,87)$ & ns \\
\hline $\begin{array}{l}\text { Foi levada a conhecer as salas de } \\
\text { parto quando internou }\end{array}$ & & & & 0,4969 & & \\
\hline Sim & $179(57,7)$ & $166(92,7)$ & $13(7,3)$ & & 1,00 & \\
\hline $\begin{array}{l}\text { Não oportunizaram/não quis/não } \\
\text { deu tempo }\end{array}$ & $131(42,25)$ & $124(94,7)$ & $7(5,3)$ & & $0,72(0,28 ; 1,86)$ & ns \\
\hline $\begin{array}{l}\text { Respeitaram a posição escolhida } \\
(n=209)\end{array}$ & & & & 0,0804 & & \\
\hline Sim & $168(80,4)$ & $162(96,4)$ & $6(3,6)$ & & 1,00 & \\
\hline Não & $41(19,6)$ & $37(90,2)$ & $4(9,8)$ & & $2,92(0,78 ; 10,87)$ & ns \\
\hline $\begin{array}{l}\text { Teve contato imediato com o } \\
\text { recém-nascido }\end{array}$ & & & & $0,3992^{\ddagger}$ & & \\
\hline Sim & $283(91,3)$ & $266(94,0)$ & $17(6,0)$ & & 1,00 & \\
\hline $\mathrm{Não}^{\dagger}$ & $27(8,7)$ & $24(88,9)$ & $3(11,1)$ & & $1,96(0,53 ; 7,15)$ & ns \\
\hline $\begin{array}{l}\text { Teve acompanhante durante o } \\
\text { trabalho de parto }\end{array}$ & & & & $1,0000^{\ddagger}$ & & \\
\hline Sim & $284(91,6)$ & $265(93,3)$ & $19(6,7)$ & & 1,00 & \\
\hline $\begin{array}{l}\text { Não quis/acompanhante não } \\
\text { disponível }\end{array}$ & $26(8,4)$ & $25(96,2)$ & $1(3,8)$ & & $0,56(0,07 ; 4,34)$ & ns \\
\hline Teve acompanhante no parto & & & & $0,4888^{\ddagger}$ & & \\
\hline Sim & $270(87,1)$ & $251(93,0)$ & $19(7,0)$ & & 1,00 & \\
\hline $\begin{array}{l}\text { Não quis/acompanhante não } \\
\text { disponível }\end{array}$ & $40(12,9)$ & $39(97,5)$ & $1(2,5)$ & & $0,34(0,04 ; 2,60)$ & ns \\
\hline $\begin{array}{l}\text { Teve acompanhante logo após o } \\
\text { parto }\end{array}$ & & & & $0,1602^{\ddagger}$ & & \\
\hline Sim & $288(92,9)$ & $271(94,1)$ & $17(5,9)$ & & 1,00 & \\
\hline $\begin{array}{l}\text { Não quis/acompanhante não } \\
\text { disponível }\end{array}$ & $22(7,1)$ & $19(86,4)$ & $3(13,6)$ & & $2,52(0,68 ; 9,35)$ & ns \\
\hline
\end{tabular}

Durante o período puerperal, no Alojamento Conjunto, as usuárias se sentiram acolhidas pela equipe e pelos estudantes $(92,5 \%)$, além de obterem respostas aos questionamentos realizados $(98,7 \%)$. Também foram orientadas a respeito dos cuidados com o $\mathrm{RN}(97,1 \%)$ e orientadas e apoiadas na prática do aleitamento $(98,4 \%)$ (Tabela 4$)$.

Foram unânimes em avaliar que o Alojamento Conjunto propiciou a formação do apego com seus recém-nascidos $(99,6 \%)$ e que o acompanhante teve oportunidade de cuidar do bebê $(77,7 \%)$. A grande maioria considerou que o tempo de internação foi suficiente para fortalecer a habilidade necessária para cuidar do filho $(97,4 \%)$ e para sanar dúvidas $(94,2 \%)$. Expressaram também que no momento da alta se sentiam preparadas e confiantes (94,8\%) (Tabela 4).

Quanto à satisfação das usuárias com o atendimento durante o puerpério, houve associação significativa com o apoio emocional recebido pelos profissionais/acadêmicos $(p=0,0389)$ e com as informações recebidas pelos mesmos $(p=0,0066)$, sendo que, quando essas ações foram realizadas parcialmente, aumentou a chance de insatisfação em 6,34 (IC95\%: 1,20; 33,43) e 33,00 (IC95\%: 4,17; 261,30) vezes, respectivamente. A chance de insatisfação também aumentou quando a mulher considerou que o tempo de internação foi parcialmente suficiente para sanar suas dúvidas $(7,85$; IC95\%: 1,$69 ; 36,48)$ e quando sentiu-se parcialmente preparada para voltar para casa $(7,16$; IC95\%:1,56; 32,82) (Tabela 4). 
Tabela 4 - Atendimento durante o puerpério e satisfação segundo as mulheres usuárias. FlorianópolisSC, $2009(\mathrm{n}=310)$

\begin{tabular}{|c|c|c|c|c|c|}
\hline Variáveis & $\begin{array}{c}\text { Total } \\
310 \\
\mathrm{n}(\%)\end{array}$ & $\begin{array}{l}\text { Satisfeita } \\
299(96,5) \\
\mathrm{n}(\%)\end{array}$ & $\begin{array}{l}\text { Insatisfeita } \\
11(3,5) \\
n(\%)\end{array}$ & Valor $\mathbf{p}^{\dagger}$ & OR(IC95\%) \\
\hline $\begin{array}{l}\text { Recebeu apoio emocional dos } \\
\text { profissionais/acadêmicos }\end{array}$ & & & & 0,0389 & \\
\hline Sim & $287(92,5)$ & $279(97,2)$ & $8(2,8)$ & & 1,00 \\
\hline Não & $10(3,3)$ & $9(90,0)$ & $1(10,0)$ & & $3,88(0,44 ; 34,36)$ \\
\hline Em parte & $13(4,2)$ & $11(84,6)$ & $2(15,4)$ & & $6,34(1,20 ; 33,43)$ \\
\hline $\begin{array}{l}\text { Profissionais/ acadêmicos deram as } \\
\text { informações solicitadas }\end{array}$ & & & & 0,0066 & \\
\hline Sim & $306(98,7)$ & $297(97,1)$ & $9(2,9)$ & & 1,00 \\
\hline Em parte & $4(1,3)$ & $2(50,0)$ & $2(50,0)$ & & $33,00(4,17 ; 261,30)$ \\
\hline $\begin{array}{l}\text { Recebeu orientação sobre cuidados com } \\
\text { o recém-nascido }\end{array}$ & & & & 0,2818 & \\
\hline Sim & $301(97,1)$ & $291(96,7)$ & $10(3,3)$ & & 1,00 \\
\hline Não & $2(0,6)$ & $2(100,0)$ & $0(0,0)$ & & $5,55(0,25 ; 123,03)$ \\
\hline Em parte & $7(2,6)$ & $6(85,7)$ & $1(14,3)$ & & $6,41(0,98 ; 41,95)$ \\
\hline $\begin{array}{l}\text { Recebeu orientação e apoio sobre } \\
\text { aleitamento materno }\end{array}$ & & & & 1,0000 & \\
\hline Sim & $305(98,4)$ & $294(96,4)$ & $11(3,6)$ & & 1,00 \\
\hline Não & $3(1,0)$ & $3(100,0)$ & $0(0,0)$ & & $3,66(0,18 ; 75,06)$ \\
\hline Em parte & $2(0,6)$ & $2(100,0)$ & $0(0,0)$ & & $5,12(0,23 ; 112,90)$ \\
\hline $\begin{array}{l}\text { Alojamento conjunto favoreceu } \\
\text { vinculação afetiva com o recém-nascido }\end{array}$ & & & & 1,0000 & \\
\hline Sim & $309(99,6)$ & $298(96,4)$ & $11(3,6)$ & & 1,00 \\
\hline Não & $1(0,4)$ & $1(100,0)$ & $0(0,0)$ & & $8,65(0,33 ; 224,13)$ \\
\hline $\begin{array}{l}\text { Acompanhante participou dos cuidados } \\
\text { com o recém-nascido* }\end{array}$ & & & & 0,2112 & \\
\hline Sim & $241(77,7)$ & $235(97,5)$ & $6(2,5)$ & & 1,00 \\
\hline Não & $37(11,9)$ & $35(94,6)$ & $2(5,4)$ & & $2,55(0,57 ; 11,44)$ \\
\hline Em parte & $30(9,7)$ & $28(93,3)$ & $2(6,7)$ & & $3,18(0,70 ; 14,38)$ \\
\hline $\begin{array}{l}\text { Tempo de internação suficiente para } \\
\text { desenvolver confiança/capacidade de } \\
\text { cuidar do recém-nascido }\end{array}$ & & & & 0,2574 & \\
\hline Sim & $302(97,4)$ & $292(96,7)$ & $10(3,3)$ & & 1,00 \\
\hline Não & $1(0,4)$ & $1(100,0)$ & $0(0,0)$ & & $9,29(0,36 ; 241,73)$ \\
\hline Em parte & $7(2,2)$ & $6(85,7)$ & $1(14,3)$ & & $6,43(0,98 ; 42,09)$ \\
\hline $\begin{array}{l}\text { Tempo de internação suficiente para } \\
\text { sanar dúvidas }\end{array}$ & & & & 0,0683 & \\
\hline Sim & $292(94,2)$ & $283(96,9)$ & $9(3,1)$ & & 1,00 \\
\hline Não & $7(2,2)$ & $7(100,0)$ & $0(0,0)$ & & $1,99(0,11 ; 37,44)$ \\
\hline Em parte & $11(3,6)$ & $9(81,8)$ & $2(18,2)$ & & $7,85(1,69 ; 36,48)$ \\
\hline Sentiu preparada para voltar para casa & & & & 0,0952 & \\
\hline Sim & $294(94,8)$ & $285(96,9)$ & $9(3,1)$ & & 1,00 \\
\hline Não & $4(1,3)$ & $4(100,0)$ & $0(0,0)$ & & $3,34(0,17 ; 66,56)$ \\
\hline Em parte & $12(3,9)$ & $10(83,3)$ & $2(16,7)$ & & $7,16(1,56 ; 32,82)$ \\
\hline
\end{tabular}

${ }^{*}$ Duas mulheres não tiveram acompanhante; ${ }^{\dagger}$ Teste exato de Fisher.

\section{DISCUSSÃO}

A população estudada apresentou características sociodemográficas similares ao perfil apresentado em outras pesquisas desenvolvidas com mulheres nesta mesma maternidade ${ }^{9}$ e em investigações realizadas em outras instituições públicas brasileiras, ${ }^{3,9}$ ou seja, são jovens com pouca escolaridade e com necessidades sociais explícitas. Representam, pois, o contingente de mulheres em idade reprodutiva que procuram os serviços de 
saúde do SUS. Os dados obstétricos se coadunam com algumas características apontadas em publicações nacionais. ${ }^{10-11}$ No entanto, se diferenciam com relação ao número de partos na posição vertical, provavelmente este resultado seja reflexo da filosofia assistencial da maternidade, que viabiliza, dentre outras ações, a adoção de posições que a própria mulher entenda ser melhor para parir., ${ }^{9,12-14}$

Quanto ao atendimento recebido na admissão obstétrica, as usuárias avaliaram que os profissionais e os acadêmicos se preocuparam em se identificar e estavam interessados em recebê-las na unidade. Isso denota a operacionalização dos princípios filosóficos da maternidade, voltados para atitudes de respeito à clientela que procura o serviço. Se por um lado tem-se em mente que atitudes como essa não deveriam ser prerrogativas deste ou daquele profissional, ou desta ou daquela unidade assistencial - já que não identificar-se e não mostrar-se atencioso seriam ações contrárias às agendas ditas de "humanização", ${ }^{15-16}$ por outro, sabe-se que não são poucos os resultados investigativos que constatam a ausência de condutas nesse sentido, particularmente nos cenários assistenciais voltados ao parto em instituições públicas e, mais especificamente ainda, em hospitais de ensino. ${ }^{17-19}$

$\mathrm{O}$ fato de perceberem que os profissionais estavam preparados para o atendimento e que não foram submetidas a práticas comprovadamente danosas ou inefetivas, ${ }^{20}$ como o enema e a tricotomia, leva-nos a inferir que a qualificação contínua da equipe é outro princípio que está sendo operacionalizado. Aliás, a efetivação desse princípio é reveladora de que profissionais e acadêmicos de enfermagem e medicina atingiram proficiência nas habilidades necessárias para manejar a gestação normal. ${ }^{21}$ Os achados também apontam que, na admissão, as mulheres se sentiram acolhidas, tanto por receber as informações desejadas, quanto por terem ao lado o acompanhante de sua escolha. Esses resultados mostram que os princípios relacionados com os direitos dos usuários estão sendo implementados já no momento da internação.

Com relação ao trabalho de parto/parto, as usuárias avaliaram que os princípios filosóficos continuaram sendo colocados em prática em sua maioria, uma vez que tiveram a privacidade respeitada e sentiram-se apoiadas do ponto de vista emocional e informacional durante todo o processo do nascimento, inclusive com direito ao acompanhamento da pessoa de sua confiança. Esses achados também revelam que a Lei n. 11.108, ${ }^{22}$ conhecida como "Lei do acompanhante" está sendo implementada na maternidade estudada e são semelhantes a alguns estudos, ${ }^{11-12}$ mas discrepantes de outros realizados em instituições de saúde. .7,23-24 $^{17}$

No trabalho de parto, especificamente, as mulheres destacaram a realização de condutas obstétricas condizentes com as evidências científicas e as recomendações da $\mathrm{OMS}^{20}$ (deambulação, movimentação e uso de métodos não farmacológicos para alívio da dor). A adoção dessas práticas ainda não é observada em outras maternidades brasileiras, ${ }^{23-24}$ o que reafirma a contribuição de uma filosofia que norteie o processo assistencial. No entanto, chama atenção que o número de mulheres com restrição alimentar excedeu às submetidas à cesariana, revelando a necessidade de identificar os fatores determinantes dessa conduta que também tem sido praticada em várias maternidades do país. ${ }^{11,17,23} \mathrm{O}$ fato de poderem escolher a posição para parir e de terem essa posição respeitada pela equipe, além do contato imediato com o filho, logo após o parto, são indicadores positivos da concretização da maioria dos princípios filosóficos também durante o parto e o pós-parto imediato. Desta forma, pode-se inferir que a equipe de saúde, ao incorporar e praticar princípios que possibilitem que a mulher participe das decisões, contribui para a consolidação de práticas comprovadamente úteis na atenção ao parto ${ }^{20}$ à exemplo do parto na posição vertical, que não tem sido adotada em maternidade públicas. ${ }^{17,24}$

O período do puerpério, apesar de ser vivenciado no Alojamento Conjunto, que é uma unidade totalmente diversa em termos de localização, de composição da equipe e de tempo de permanência, se comparada ao Centro Obstétrico, também teve avaliação similar no que se refere ao atendimento recebido. Contudo, como esperado, as mulheres destacaram as orientações recebidas com relação aos cuidados com o RN, a prática do aleitamento materno e o envolvimento do acompanhante nos cuidados.

Chamou atenção a apreciação altamente positiva acerca do tempo de internação como um aspecto favorecedor do preparo da mulher para a alta, no que se refere ao desenvolvimento da confiança para cuidar do filho e resolução de dúvidas. Tal avaliação coaduna-se com a implementação dos princípios filosóficos voltados para a compreensão do ciclo gravídico-puerperal como um processo e não apenas como um evento isolado, a flexibilização das rotinas assistenciais, o tempo de internação relacionado com as necessidades individuais da clientela assistida, a importância da participação da família e a valorização do Alojamento Conjunto 
como sistema que propicia o acompanhamento sistemático e contínuo da saúde do binômio, o desenvolvimento de atividades educativas, bem como a promoção do vínculo e interação familiar.

Este resultado sobre a valorização do Alojamento como um espaço de educação em saúde e de interação entre os integrantes da família, tem sido amplamente confirmado na literatura nacional, $11,17,19$ enquanto os demais aspectos avaliados positivamente pelas usuárias, não têm sido alvo de avaliação sistemática em outros estudos. Esses achados demonstram que a filosofia assistencial está sendo colocada em prática no Alojamento Conjunto e seus princípios contemplam necessidades relacionadas não somente com educação em saúde e vínculos familiares, mas com aspectos mais abrangentes do período puerperal.

A satisfação das mulheres esteve associada com os aspectos envolvidos na qualidade do cuidado recebido. Destaca-se que o apoio emocional e as informações recebidas dos profissionais/ acadêmicos, em todas as etapas do processo (na admissão, durante o trabalho de parto/parto e puerpério), foram preditores desta satisfação. Por outro lado, quando esses atributos são realizados apenas de forma parcial, isso influencia fortemente na chance de insatisfação das mulheres, o que por sua vez significa que os princípios filosóficos precisam ser revitalizados na prática assistencial da maternidade estudada.

Outros fatores também influenciam na satisfação das usuárias, como o fato de os profissionais se apresentarem, estarem capacitados e demonstrarem interesse em atender, assim que a mulher dá entrada na instituição. Além disso, ter a privacidade respeitada durante o trabalho de parto/parto e permanecer internada tempo suficiente para sanar as dúvidas, sentindo-se preparada para voltar para casa, são fatores que também contribuem para a satisfação.

Tais resultados se coadunam com estudos desenvolvidos acerca da satisfação de mulheres com o parto, que mostram que critérios como a boa comunicação, a qualidade do relacionamento entre profissionais de saúde e parturientes, e a suficiente provisão de informações e cuidados são vitais para que a avaliação seja positiva. ${ }^{6,8,25}$ Ensaio clínico randomizado controlado sobre a satisfação com a experiência do nascimento, por exemplo, apontou resultados semelhantes no que se refere à satisfação das usuárias com as informações recebidas dos profissionais, ${ }^{8}$ reforçando a influência positiva desse aspecto na avaliação realizada pelas mulheres. Em contrapartida, a maioria dos estudos mostra que, de maneira geral, há insatisfação com relação às informações recebidas da equipe de saúde em outras realidades assistenciais. ${ }^{12,24-26}$

\section{CONCLUSÕES}

A operacionalização dos princípios filosóficos foi avaliada positivamente pelas mulheres, o que resultou em elevado grau de satisfação. Conclui-se que a existência e a concretização de uma filosofia assistencial contribuem para a satisfação das usuárias, desde a internação até a alta.

$\mathrm{O}$ fato dessas mulheres terem sido entrevistadas ainda durante a internação, pode ter se constituído numa limitação do estudo, uma vez que as respostas das mesmas com relação à satisfação sobre o atendimento recebido podem ter sido influenciadas pelo contexto assistencial. No entanto, a maioria delas expressou que não foi submetida a práticas obstétricas desnecessárias, o que mostra que a atenção obstétrica está ancorada nas evidências científicas, no respeito às escolhas informadas e no estímulo à inclusão do acompanhante.

Considerando-se que o estudo foi desenvolvido em um hospital que é centro formador de recursos humanos em saúde, esses achados apontam para os benefícios que a concretização de uma filosofia pode ter, não apenas para a assistência aos usuários, mas também para o processo de formação, funcionando como exemplo prático para que os acadêmicos da área da saúde tomem esse modelo como referência de atuação profissional futura.

\section{REFERÊNCIAS}

1. Monticelli M, Brüggemann OM, Guerini IC, Boing AF, Padilha MF, Fernandes VB. A filosofia assistencial da maternidade de um hospital universitário na visão dos acadêmicos. Texto Contexto Enferm 2010 Jan-Mar; 19 (1):25-35.

2. World Health Organization. Appropriate technology for birth. Lancet 1985 Aug 24; 2(8452):436-7.

3. Carr KC. Developing and evidence-based practice protocol: implications for midwifery practice. J Miidwifery Womens Health. 2000 Nov-Dec; 45(6):544-51.

4. Santos OMB, Siebert ERC. The humanization of birth experience at the University of Santa Catarina maternity hospital. Int J Gynecol Obstet. 2001 Nov; 75(Suppl 1):S73-9.

5. Guerini IC, Monticelli M, Brüggemann OM. A filosofia da maternidade do HU/UFSC na visão dos usuários, profissionais e acadêmicos: nota prévia. Online Braz J Nurs [online]. 2009 [acesso 
2010 Ago 19]; 8:[3p.]. Disponível em: http://www. objnursing.uff.br/index.php/nursing/article/ view/j.1676-4285.2009.1959/439

6. Martin $\mathrm{CH}$, Fleming V. The birth satisfaction scale. Int J Health Care Qual Assur. 2011 [Cited 2011 Ago 19]; 24(2):124-35. Available from: http:/ / emeraldinsight. com/journals.htm?issn $=0952-6862 \&$ volume $=24 \&$ iss ue $=2$ \&articleid $=1906029 \&$ show $=\mathrm{html}$

7. Brown S, Lumley J. Satisfaction with care in labor and birth: as survey of 790 Australian women. Birth 1994 Mar; 21(1):4-13.

8. Brüggemann OM, Parpinelli MA, Osis MJD, Cecatti JG, Carvalhinho Neto AS. Support to woman by a companion of her choice during childbirth: a randomized controlled trial. Reprod Health [online]. 2007 Jul 6 [cited 2010 Jun 30]; 4:. Available from: http:/ / www.reproductive-health-journal.com/ content/4/1/5.

9. Santos PQ, Pinheiro CED, Souza ML, Santos ML, Monticelli M, Diniz CSG. Maternal position at birth and the newborn Apgar score: cross-sectional study. Online Braz J Nurs [online]. 2009 [cited 2010 Mai 06]; 8:[10 p.]. Available from: http://www. objnursing.uff.br/index.php/nursing/article/ view/j.1676-4285.2009.2517/html_40.

10. Nagahama EEI, Santiago SM. Humanização e equidade na atenção ao parto em município da região sul do Brasil. Acta Paul Enferm 2008; 21(4): 609-15.

11. Simões SMF, Jesus DV, Boechat JS. Childbirth and assistance: a quantitative study. Online Braz J Nurs [online]. 2007 [cited 2010 Mai 20]; 6(2): [14 p.]. Available from: http://www. objnursing.uff.br/index.php/nursing/article/ view/j.1676-4285.2007.879/211.

12. Nagahama EEI, Santiago SM. Práticas de atenção ao parto e os desafios para humanização do cuidado em dois hospitais vinculados ao Sistema Único de Saúde em município da Região Sul do Brasil. Cad Saúde Pública 2008 Ago; 24(8):1859-68.

13. Brüggemann OM, Knobel R, Siebert ERC, Boing AF, Andrezzo KFA Parto vertical em hospital Universitário: série histórica, 1996 a 2005. Rev Bras Saúde Matern Infant. 2009 Abr-Jun; 9(2):189-96.

14. Basso JF, Monticelli M. Expectations of pregnant women and partners concerning their participation in humanized births. Rev Latino-am Enfermagem 2010 May-Jun; 18(3):390-7.

15. Machado NXS, Praça NS. Centro de parto normal e assistência obstétrica centrada nas necessidades da parturiente. Rev Esc Enferm USP. 2006 Jun; 40(2):274-9.

16. Campos SEV, Lana FCF. Resultados da assistência ao parto no Centro de Parto Normal Dr. David Capistrano da Costa Filho em Belo Horizonte, Minas Gerais, Brasil. Cad Saúde Pública. 2007 Jun; 23(6):1349-59.

17. Reis AE, Patrício ZM. Aplicação das ações preconizadas pelo Ministério da Saúde para o parto humanizado em um hospital de Santa Catarina. Ciênc Saúde Coletiva. 2005 Sep-Dec; 10(Sup):221-30.

18. Hotimsky SN, Rattner D, Vanancio SI, Bógus CM, Miranda MM. O parto como eu vejo... ou como eu o desejo? expectativas de gestantes, usuárias do SUS, acerca do parto e da assistência obstétrica. Cad Saúde Pública. 2002 Set-Out; 18(5):1303-11.

19. Tronchin DMR, Melleiro MM, Tsunechiro MA, Gualda DMR. O olhar dos usuários de um hospital de ensino: uma análise da qualidade assistencial às gestantes e aos recém-nascidos. Texto Contexto Enferm. 2006 Jul-Set; 15(3):401-8.

20. World Health Organization. Care in normal birth: a practical guide. Geneva (CH): WHO; 1996.

21. Word Health Organization. Making pregnancy safer - the critical role of the skilled attendant: a joint statement by WHO, ICM, and FIGO. Geneva (CH): WHO; 2004.

22. Brasil. Lei n. 11.108, de 7 de abril de 2005: altera a Lei n. 8.080, de 19 de setembro de 1990, para garantir às parturientes o direito à presença de acompanhante durante o trabalho de parto, parto e pós-parto imediato, no âmbito do Sistema Único de Saúde SUS. Diário Oficial da União. 8 Abr 2005; Seção 1:1.

23. Manzini FC, Borges VTM, Parada CMGL. Avaliação da assistência ao parto em maternidade terciária do interior do Estado de São Paulo, Brasil. Rev Bras Saúde Matern Infant. 2009 Jan-Mar; 9(1):59-67.

24. D'Orsi E, Chor D, Giffin K, Ângulo-Tuesta A, Barbosa GP, Gama AS, et al. Qualidade da atenção ao parto em maternidades do Rio de Janeiro. Rev Saúde Pública. 2005 Ago; 39(4):646-54.

25. Hodnet ED. Pain and women's satisfaction with the experience of childbirth: a sistematic review. Am J Obstet Gynecol. 2002 May; 186(5 Suppl):S160-72.

26. Queiroz MVO, Jorge MSB, Marques JF, Cavalcante AM, Moreira KAP. Indicadores de qualidade da assistência ao nascimento baseados na satisfação de puérperas. Texto Contexto Enferm. 2007 Jul-Set; 16(3):479-87. 